Treatment of Staphylococcal Infections

\title{
TREATMENT OF GENERALIZED STAPHYLOCOCCAL INFECTIONS
}

\author{
William M. M. KIRBY \\ Department of Medicine, \\ University of Washington School of Medicine, Seattle, Washington.
}

The principal contribution of the new penicillins has been in the therapy of severe generalized infections caused by staphylococci resistant to penicillin G. Considering the overall aspects of efficacy and side effects, experience for more than five years has demonstrated the superiority of methicillin over previous antibiotics including vancomycin, and the combination of erythromycin and chloramphenicol. From 1950 to 1957 , the cure rate in patients with staphylococcal bacteraemia with a variety of drugs, chiefly erythromycin and chloramphenicol, was around $40 \%$ or lower. (Wilson and Hamburger, 1957; Geraci, Hoffmeister - Nichols and Needham, 1958; Hassall and Rountree, 1959). Considerable improvement was noted with vancomycin, a $65 \%$ survival rate being reported among 121 cases (Kirby, 1963). With methicillin the survival rate has been around $70 \%$ in over 200 cases, (Klein and Finland, 1963; Allen, Roberts and Kirby, 1962), a remarkable figure considering the high incidence of serious underlying illnesses and the frequent inaccessibility of major foci of infection. The importance of the latter point was stressed in one report, where five of six patients who received optimal drainage of a local lesion survived, while in 13 patients where suitable drainage was not possible, ten died despite adequate methicillin therapy (Sabath, Postic and Finland, 1962).

Similar results, i.e., a mortality rate of $30 \%$ or less, have been reported in serious staphylococcal infections in nonbacteraemic patients, and it is possible that even better figures might have been attained with larger doses of methicillin. Since the minimum inhibitory concentration is 3 to $4 \mu \mathrm{g}$./ $/ \mathrm{ml}$. for a large inoculum of most strains, and the peak serum concentration is 12 to $14 \mu \mathrm{g} . / \mathrm{ml}$. following an intramuscular injection of 1 gram, it is probable that 2 grams should be administered every 4 hours for optimal penetration into infected tissues. Gold (1962) noted positive blood cultures in two cases treated with
6 grams per day, with clearing of the bacteraemia only after the dose was doubled. The importance of large doses has also been recognized by other investigators (Klein and Finland, 1963; Allen, Roberts and Kirby, 1962).

Initial studies performed five years ago led some of us to predict that emergence of methicillin-resistant staphylococci would not become a problem of appreciable clinical significance (Kirby, 1961). This prediction, which now appears to have been correct, was based on finding the same slow, stepwise development of resistance in vitro to methicillin as was noted with penicillin G, since in patients staphylococci have not become ree sistant to penicillin $G$ through this mechanism Also, the incidence of naturally-occurring methicillin-resistant strains was very low, in contrast to the significant numbers of penicie linase producers observed with the earliest studies of penicillin G (Kirby, 1945). Superinfection with gram-negative bacilli has been a frequent complication of methicillin therapy, particularly in debilitated patients in charity hospitals (Sabath, Postic and Finland, 1962). This is in no way specific for methicillin, but represents the change in flora noted previously with other antibiotics bactericidal for most gram positive organisms (Kirby, 1963). As for allergic reactions, it is now apparent that there is cross-sensitization between all the penicillins. A low incidence of bone marrow depression and nephrotoxicity has also been attributed to methicillin. As with penicillin G, however, the great majority of patients have tolerated the new penicillins with a minimal incidence of toxicity and side effects.

Since the advent of methicillin, a number of other penicillinase-resistant synthetic penicillins have been used clinically, including oxacillin, cloxacillin, diphenicillin, dicloxacillin, and nafcillin. Although better absorbed following oral administration, none of these new compounds has shown clearcut superiority over methicillin from the standpoint of clinical efficacy. Since clinical comparisons are notori- 
ously difficult with staphylococcal infections, other factors are helpful in assessing efficacy, including relative in vitro activity, absorption and excretion, and serum binding.

Oxacillin, 5 to 8 times as active as methicillin against staphylococci in vitro, is well absorbed following oral administration, and these advantages would seem sufficient to make methicillin obsolete. On the other hand, oxacillin is bound by serum proteins to a much greater extent than methicillin (80 to $90 \%$ compared with less than $20 \%$ ) and this feature probably cancels out the advantage of its greater antibacterial activity. The clinical significance of serum binding is difficult to determine, and some have doubted that it represents a disadvantage at all. However, the bound antibiotic is not active against bacteria (Kirby, 1962), and it has been shown that the concentration of free antibiotic in the tissues is essentially the same as that present in the serum (Verwey and Williams, 1962; Scholtan and Schmid, 1962). Direct measurements in patients have also shown that methicillin diffuses into peritoneal, pleural, joint, and cerebrospinal fluids in higher concentrations than oxacillin, suggesting that methicillin may be preferable for infections involving these sites (Geraci et al., 1962; Rutenberg, Greenberg and Schweinberg, 1960; White and Varga, 1961). In another type of study when serum concentrations were determined following intramuscular injections by a method combining the factors of absorption, antistaphylococcal activity, and protein binding, very similar values were obtained for methicillin (1 gram) and oxacillin ( 0.5 gram), and also for nafcillin and cephalothin, 0.5 gram each (Sidell et al., 1963). Finally, clinical results so far available do not support the superiority of its greater antistaphylococcal activity. In comparing results in patients seriously ill with staphylococcal bacteræmia and with pneumonia, Finland's group found that the mortality for those treated with methicillin and oxacillin was almost identical. Even though intramuscular and intravenous dosage forms are now available for oxacillin, it is apparent that methicillin should not be abandoned at present, and that further careful laboratory and clinical comparisons are desirable.

Cloxacillin is similar to oxacillin in serum binding, but has greater activity in vitro against staphylococci resistant to methicillin, and serum concentrations following oral administration are at least twice as high as with oxacillin (Knudsen et al., 1962; Sidell $\stackrel{\varrho}{\frac{0}{2}}$ et al., 1964). It is of interest that serum $\stackrel{ه}{\stackrel{1}{\varrho}}$ concentrations of cloxacillin are also higher $\stackrel{c}{.}$ following intramuscular administration, and $\overrightarrow{\vec{F}}$ indirect evidence indicates that this is probably $\stackrel{0}{+}$ due at least in part to more rapid destruction of $\bar{c}$ oxacillin by the liver (Gravenkemper et al., $\frac{\overline{\bar{C}}}{\mathrm{~N}}$ 1963). Clinical studies indicate that cloxacillin $\frac{\bar{D}}{\sigma}$ is at least as effective as methicillin and oxa- $\stackrel{\varnothing}{\circ}$ cillin. In a carefully conducted study in six hospitals in England, 73 of 92 patients were $\vec{\circ}$ cured or much improved (Stewart et al., 1962), but critical comparisons with results obtained $\vec{\omega}$ with the other antibiotics are not possible? due to inevitable variability in the case material. Dicloxacillin, only recently available, appears to give blood levels twice as high as those of $\overrightarrow{0}$ cloxacillin following oral administration, but clinical studies are as yet too preliminary to warrant valid conclusions. These developments make it quite likely that one of the other isoxazolyl penicillins may eventually supplant oxacillin.

Diphenicillin represents an interesting example of the correlation between laboratory and clinical results. In vitro, diphenicillin somewhat less active than oxacillin against penicillinase-producing staphylococci, and is bound by serum proteins to approximater. the same extent as oxacillin (Ábu-Nassar, 음 Williams and Yow, 1963). Serum concentrations are distinctly inferior with diphenicillin as compared with oxacillin, especially with

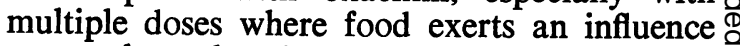
even when the drugs are taken two hours $\overrightarrow{\vec{B}}$ after meals (Kirby et al., 1962). Clinically, $\frac{\circ}{3}$ the mortality rate among patients seriously ill with staphylococcal infections who were treated with diphenicillin was appreciably greater in one series than for methicillin and 3 oxacillin (Klein, 1963b). Those treated with diphenicillin included a somewhat larger number of less favourable cases, but even $\delta$ considering this factor, the clinical results are in keeping with the in vitro and pharmacologic 옹 observations. Because of its inferior perform- $\rightarrow$ ance, as well as for certain other reasons, diphenicillin has not become available com- N mercially in the United States.

Nafcillin, which has only recently become available for large scale use, is slightly more $\mathrm{\omega}$ active in vitro, and somewhat less bound by serum proteins, than is true for oxacillin and cloxacillin. Serum concentrations of nafcillin $\stackrel{\mathbb{P}^{+}}{+}$ and oxacillin are approximately the same? following intramuscular injection, but nafcillin 
gives somewhat lower blood levels following oral administration, even with the buffered salt (Whitehouse et al., 1963; Kirby-unpublished observations). In an interesting and carefully controlled double blind study of efficacy, Martin et al. (1963), found that nafcillin gave clinical results comparable to those obtained with penicillin $G$ in penicillin treatable infections, and that nafcillin and methicillin were equally effective in the treatment of infections caused by penicillinaseproducing staphylococci. Of special interest was the fact that 14 of 36 patients with staphylococcal infections were initially misdiagnosed, and six of the seven patients who were treated with penicillin $G$ died. Of seven such patients receiving nafcillin, only one died. These figures cast serious doubt on the widely advocated practice of withholding the new penicillins until a staphylococcal aetiology is clearly established. In our opinion, a better practice is to initiate therapy with a penicillinase-resistant penicillin, and to change to penicillin $G$ in a day or two if the infection is found to be caused by susceptible staphylococci, streptococci, or pneumococci.

Apart from interest centering around the comparative clinical efficacy of these new penicillins, there are many special aspects of therapy involving seriously ill patients. One is uraemia, for patients with generalized staphylococcal infections often have renal insufficiency as one of their complications. In uraemic patients, we have made the interesting observation that oxacillin disappears from the serum much more rapidly than methicillin, and that the disappearance curves are not appreciably altered by hemodialysis (Kirby et al., 1962). Oxacillin presumably disappears more rapidly because of greater destruction by the liver, and we have recently substantiated this explanation in patients with severe liver and renal disease following ingestion of carbon tetrachloride, where the rate of disappearance of both oxacillin and methicillin is much slower.

In conclusion, it can be stated that the new penicillins have returned the therapy of severe generalized staphylococcal infections to the status that existed when penicillin $G$ was first developed. Indeed, the situation is even more favourable since the emergence of resistant strains has so far been a negligible problem. Problems for the immediate future include delineation of the relative efficacy of the several penicillinase-resistant penicillins now available, since it seems scarcely necessary to have half a dozen compounds which are essentially identical in their efficacy. There is also, of course, the always present hope that compounds that are even more effective, and that have fewer side effects, will be developed.

\section{REFERENCES}

Abu-NaSsar, H., Williams, T. W., Jr., and Yow, E. M. (1963), Amer. J. Med. Sci., 245, 399.

Allen, J. D., RoberTs, C. E., and KIRBY, W. M. M. (1962), New Engl. J. Med., 266, 111.

Bulger, R. J., LindHOlm. D D., MuRRAY, J. S., and KIRBY, W. M. M. (1964), J. Amer. Med. Ass., $187,319$.

GeraCI, J. E. (1950), Med. Clin. N. Am., 42, 1101.

Geraci, J. E., Hoffmeister, R. T., Nichols, D. R., and Needham, G. M. (1962), Proc. Mayo Clinic, 37, 137.

Gold, J. A. (1962), Pp. 693-696 in Antimicrobial Agents and Chemotherapy-1961. Ann Arbor: Braun-Brumfield, Inc.

GravenKemper, C. F., Sweedler, D. R., Brodie, J. L., Sidell, S., and KIRBY, W. M. M. (1964), Pp. 231-236 in Antimicrobial Agents and Chemotherapy-1693. Ann Arbor: Braun-Brumfield, Inc.

Hassall, J. E., and Rountree, P. M. (1959), Lancet, i, 213 .

KIRBY, W. M. M. (1945), J. clin. Invest., 24, 170.

Kirby, W. M. M. (1961), Pp. 75-76 in A Symposium on the New Dimethoxyphenyl Penicillin. Syracuse: State University of New York. P. A. Bunn, ed.

KIRBY, W. M. M. (1963), Pp. 84-96 in the Proceedings of the Second International Symposium of Chemotherapy. Naples

Kirby, W. M. M., Rosenfeld, L. S., and Brodie, J. (1962a), J. Amer. med. Ass., 181, 739.

Kirby, W. M. M., Allen, J. D., Rosenfeld, L. S., and Hull, P. Q. (1962b), Pp. 757-764 in Antimicrobial Agents and Chemotherapy-1961. Ann Arbor: Braun-Brumfield, Inc.

Klein, J. O., Sabath. L. D., Steinhauer, B. W., and FinLaND, M. (1963a), New Engl. J. Med., 269, 1215.

Klein, J. O., Sabath. L. D., Steinhauer, B. W., and FinLAND, M. (1963), Amer. J. Med. Sci., 246, 385.

KLEIN, J. O., and FinLAND, M. (1963), New Engl. J. Med., 269, 1019, 1074, 1129.

KNudSEN, E. T., Brown, D. M., and Rolinson, G. N. (1962), Lancet ii, 632.

Martin, C. M., Kushnick, T. H., Nuccio, P. A., Gray, D. F., BERNSTEIN, I., and WebB, C., Jr. Pp. 290-298 in Antimicrobial Agents and Chemotherapy-1963. Ann Arbor: Braun-Brumfield, Inc.

Rutenberg. A. M.. Greenberg, H. L., and SchweinBERG, F. B. (1960), New Engl. J. Med., 263, 1174.

Sabath, L. D., Postic, B . ant Finland, M. (1962), New Engl. J. Med., 267, 1049.

Scholtan, W., and Schmid, J. (1962), ArzneimittelForsch., 12, 741.

Sidell, S., Burdick, R. E., Brodie, J., Bulger, R. J., and KIRBY, W. M. M. (1963), Arch. intern. Med., 112, 21 .

Sidell, S., Bulger. R. J., Brodie, J. L., and Kirby, W. M. M. (1964), Clin. Pharmad. Ther., 5, 26.

STEWART, G. T., ed. (1962), Lancet, ii, 634. 
Verivey, W. F., and Williams, H. R., Jr. (1963), Pp. 476-483 in Antimicrobial Agents and Chemotherapy-1962. Ann Arbor: Braun-Brumfield, Inc.

White, A., and Varga, D. (1961), Arch. intern. Med., 108, 671 .
Whitehouse, A. C., Morgan, J. G., Schumacher, JK and Hamburger, M. (1963), Pp. 304-392 in Ant microbial Agents and Chemotherapy-1962. Ann Arbor: Braun-Brumfield, Inc.

Wilson, R., and HAMBurger, M. (1957), Amer. Med., 22, 437.

\title{
PENICILLIN THERAPY IN RELATION TO CHEST DISEASES
}

\author{
C. A. Green \\ Department of Microbiology \\ The Royal Victoria Infirmary and the University, Newcastle-upon-Tyne.
}

THE term chest infection is a wide one and in one sense there is no need to discuss penicillin therapy in relation to chest infection. Thus classical lobar pneumonia is a rarity in hospital in these days and is regarded by the present generation of medical students as a clinical curio. The clinical bacteriologist in his laboratory has come to regard colonies of Streptococcus pneumoniae as being of relatively little importance, since he knows full well that by the time he has cultured the organism, the clinician will have treated the patient with some form of penicillin and that in all probability, the clinical condition will have resolved. So much so that an organism alleged to be a pneumococcus which is resistant to penicillin would be regarded with suspicion and in fact it is almost unknown in our laboratory to find such an organism. Thus therapy for conditions such as lobar pneumonia presents no problem. Penicillin $G$ or one of its analogues is given and rarely does the condition progress to the classical picture.

Ours is a general hospital which also functions as the teaching hospital for the University of Newcastle-upon-Tyne. Since 1950 we have made it our standard practice to examine the sensitivity of all pathogens isolated, to a reasonably wide range of antibiotics. From our records Dr. A. J. Wort has constructed two tables. In late 1950 and 1951 we did not record the diagnoses or sources of the organisms which we isolated and the original requests inevitably are no longer available.

We are not, therefore, able to show the pattern of sensitivity at this time in relation to chest infection. I thought it, however, fair to make certain deductions from the available data. Table I shows that in the winter period 1950-1951 we isolated a total of 232 staphylococci and of these $132(57 \%)$ weice designated penicillin resistant. I confess that am surprised by this because I would have thought that penicillin resistance had not bet come a problem of this magnitude. Howeve? the comparative figure for a similar number of organisms studied in 1962 as part of our ear work with orbenin revealed an increase up to $73 \%$ penicillin $\mathrm{G}$ resistant strains.

More recently in 1957 we introduced hhe practice of recording in our sensitivity reegrd books the diagnoses and an indication of the source of the organisms which had been isolated. From that time we have been able to estimate the incidence of staphylococcal chest infections. Table II shows the pattern of even for the period, November-February inclusi for the past seven years. We have found that the post-operative chest infection, which commonly due to a hospital staphylococcus, is a serious problem and roughly $80 \%$ of these staphylococci are resistant to penicillin $\mathrm{G}$ : This, of course, is not to say that other organ isms do not on occasions become involved ang in common with other workers we have found that Proteus spp. and Ps. pyocyanea are diffis cult to control. Nevertheless the staphylococcus is our chief problem since it appears to be se easily transferred from one patient to anothe and does so easily colonize the nasopharyn of both patients and staff alike. Many of the staphylococci which we have considered are resistant to a wide range of antibiotics, usually being sensitive only to erythromycin, novos biocin and chloramphenicol. Our clinical cob leagues are not very enthusiastic about using chloramphenicol and this effectively limits the choice of antibiotic. The release of methicillief in 1960 was, therefore, a real advance in therapy and it rapidly became the drug of 